\title{
The mercury resistance operon: from an origin in a geothermal environment to an efficient detoxification machine
}

\author{
Eric S. Boyd ${ }^{1 *}$ and Tamar Barkay ${ }^{2}$ \\ ${ }^{1}$ Department of Chemistry and Biochemistry, Montana State University, Bozeman, MT, USA \\ ${ }^{2}$ Department of Biochemistry and Microbiology, Rutgers University, New Brunswick, NJ, USA
}

Edited by:

Howard Junca, Corporacion

CorpoGen, Colombia

Reviewed by:

Mei-Fang Chien, Tohoku-Gakuin

University, Japan

Jiangxin Wang, Arizona State

University, USA

*Correspondence:

Eric S. Boyd, Department of

Chemistry and Biochemistry, 103

Chemistry Research Building,

Montana State University, Bozeman,

MT 59717, USA.

e-mail: eboyd@montana.edu
Mercuric mercury $(\mathrm{Hg}[\mathrm{II}])$ is a highly toxic and mobile element that is likely to have had a pronounced and adverse effect on biology since Earth's oxygenation $\sim 2.4$ billion years ago due to its high affinity for protein sulfhydryl groups, which upon binding destabilize protein structure and decrease enzyme activity, resulting in a decreased organismal fitness. The central enzyme in the microbial mercury detoxification system is the mercuric reductase (MerA) protein, which catalyzes the reduction of $\mathrm{Hg}(\mathrm{II})$ to volatile $\mathrm{Hg}(0)$. In addition to MerA, mer operons encode for proteins involved in regulation, $\mathrm{Hg}$ binding, and organomercury degradation. Mer-mediated approaches have had broad applications in the bioremediation of mercury-contaminated environments and industrial waste streams. Here, we examine the composition of 272 individual mer operons and quantitatively map the distribution of mer-encoded functions on both taxonomic SSU rRNA gene and MerA phylogenies. The results indicate an origin and early evolution of MerA among thermophilic bacteria and an overall increase in the complexity of mer operons through evolutionary time, suggesting continual gene recruitment and evolution leading to an improved efficiency and functional potential of the Mer detoxification system. Consistent with a positive relationship between the evolutionary history and topology of MerA and SSU rRNA gene phylogenies (Mantel $R=0.81, p<0.01$ ), the distribution of the majority of mer functions, when mapped on these phylograms, indicates an overall tendency to inherit mer-encoded functions through vertical descent. However, individual mer functions display evidence of a variable degree of vertical inheritance, with several genes exhibiting strong evidence for acquisition via lateral gene transfer and/or gene loss. Collectively, these data suggest that (i) mer has evolved from a simple system in geothermal environments to a widely distributed and more complex and efficient detoxification system, and (ii) merA is a suitable biomarker for examining the functional diversity of $\mathrm{Hg}$ detoxification and for predicting the composition of mer operons in natural environments.

Keywords: merA, mercuric reductase, trait evolution, diversity, genomics, gene loss, lateral gene transfer, operon evolution

\section{INTRODUCTION}

Mercury $(\mathrm{Hg})$ is the most toxic heavy metal due to its high affinity for the sulfhydryl ligands in amino acids, which upon binding, leads to alteration in protein structure, and often a loss of function (Nies, 2003). Two unique aspects of $\mathrm{Hg}$ geochemistry place it high on the list of potent environmental contaminants: its global distribution (Pirrone et al., 2010) and the possibility of it being converted to more toxic methylated forms (Lin et al., 2012). Methylated forms of mercury ( $\mathrm{MeHg}$ ) are produced in the environment by anaerobic bacteria and the $\mathrm{MeHg}$ that is produced is often bioaccumulated and biomagnified in aquatic (Watras et al., 1998; Boyd et al., 2009) and terrestrial (Rimmer et al., 2010) food chains, thereby posing severe consequences to human and environmental health (Clarkson and Magos, 2006). Moreover, atmospheric deposition of $\mathrm{Hg}$, coupled with methylation and biomagnification, lead to accumulation of $\mathrm{MeHg}$ in biota far removed from known sources of $\mathrm{Hg}$ (e.g., polar-regions; Dietz et al., 2009).

Some aerobic Bacteria and Archaea have evolved resistance mechanisms that function to degrade organomercury compounds and to reduce the local concentration of inorganic $\mathrm{Hg}(\mathrm{Hg}[\mathrm{II}])$ by reduction to gaseous $\mathrm{Hg}(0)$, effectively partitioning $\mathrm{Hg}(0)$ to the gaseous phase, and allowing for microbial growth (Barkay et al., 2003; Lin et al., 2012). The Hg resistance (mer) system is encoded for by the mer operon that consists of the homodimeric flavindependent disulfide oxidoreductase enzyme mercuric reductase (MerA) and which may also encode for organomercury lyase (MerB), a periplasmic $\mathrm{Hg}(\mathrm{II})$ scavenging protein (MerP), one or more inner membrane spanning proteins (MerT, MerC, MerE, MerF, MerG) that transport $\mathrm{Hg}$ (II) to the cytoplasm where it is reduced by MerA, and one or two regulatory proteins (MerR, MerD; Figure 1). The overall expression of mer is regulated by 


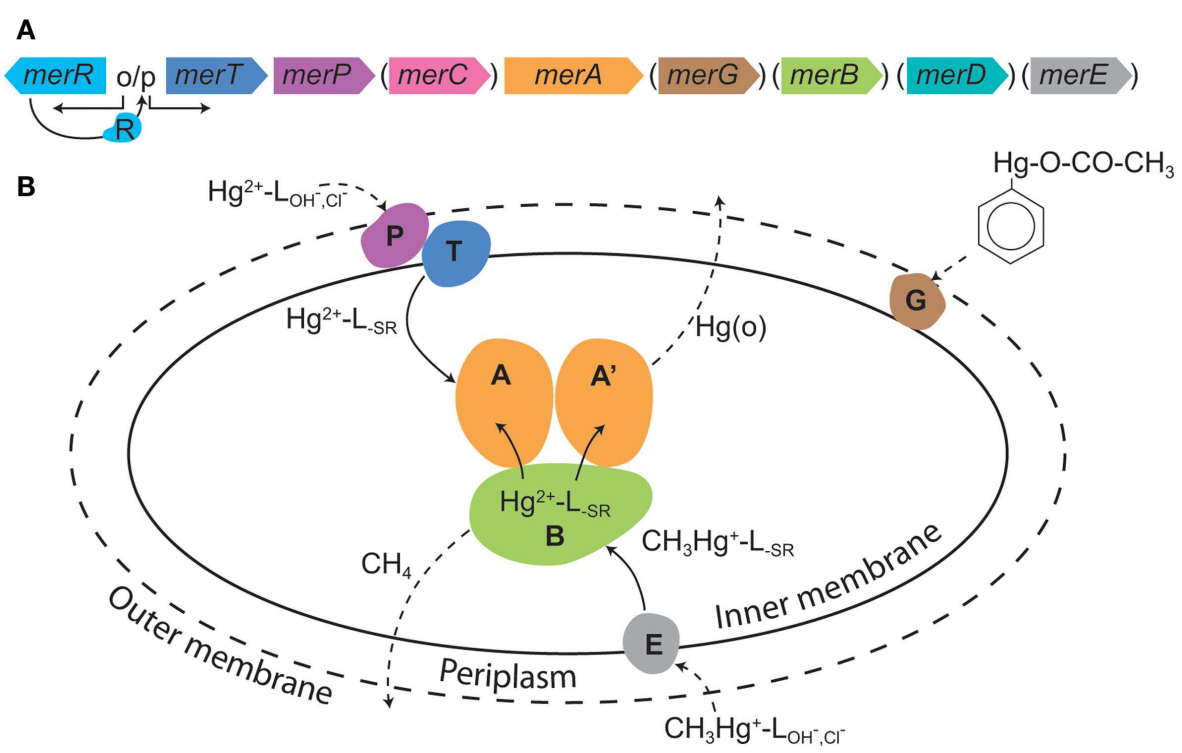

FIGURE 1 |The mer system. (A) A generic mer operon with genes in parentheses depicting those that are present in some, but not the majority of, operons. (B) The cellular mer-encoded mercury detoxification mechanisms. The outer cell wall is depicted by a broken line illustrating that not all microbes have an outer membrane; broken line arrows depict diffusion; solid line arrows indicate transport or transformations; $L=$ ligand with subscripts denoting the ligand type. The colors of various Mer proteins correspond with the colors of the genes that encode for these proteins in (A). Reproduced with permission from Lin et al. (2012).
MerR, acting as a transcriptional repressor or activator in the absence and presence of $\mathrm{Hg}(\mathrm{II})$, respectively. In addition, several proteobacteria encode for MerD which functions to down regulate operon expression (Barkay et al., 2003; Lin et al., 2012).

About 1-10\% of cultured heterotrophic, aerobic microbes from various environments possess mer systems (Barkay, 1987), alluding to the ubiquity of $\mathrm{Hg}(\mathrm{II})$ in the environment, and the high selective pressure that this imposes on microbiota to detoxify their local environment. Mercury resistant microorganisms are often enriched to even higher abundances in $\mathrm{Hg}$ contaminated environments (Barkay, 1987; Osborn et al., 1997), where their activities enhance conversion of $\mathrm{MeHg}$ (Schaefer et al., 2004) and $\mathrm{Hg}$ (II) to $\mathrm{Hg}(0)$ (Kritee et al., 2007). Furthermore, bacteria that resist Hg via the mer system have been utilized to effectively remove $\mathrm{Hg}$ from chlor-alkali bioreactor wastewater, yielding water that is suitable for disposal to municipal waste streams (Wagner-Döbler, 2003). The comprehensive understanding of mer and the functions that it encodes have facilitated the bioengineering of molecules, cells, and plants that are efficient at sorbing and/or transforming $\mathrm{Hg}$ (Lin et al., 2012). Moreover, the enhanced familiarity with the mer regulatory circuit has been exploited and used to develop highly sensitive reporters of $\mathrm{Hg}$ in environmental samples (Selifonova et al., 1993; Virta et al., 1995). Thus, the mer system is the basis for the continued development of biological tools for $\mathrm{Hg}$ bioremediation and the management of $\mathrm{Hg}$ contaminated environments.

A survey of all microbial genomes performed in 2010 greatly expanded our understanding of the diversity of microorganisms that possess the mer detoxification machinery and identified its presence among early evolving lineages of thermophilic Bacteria and Archaea. This suggested that $\mathrm{Hg}$ resistance most likely originated in a hydrothermal environment, where geochemically derived $\mathrm{Hg}$ is at a naturally elevated concentration (Barkay et al., 2010). Unlike the mer operons in the Proteobacteria, Firmicutes, and Actinobacteria, the mer operons of early evolving microbial lineages encode for fewer functional genes. The regulation of mer functions in some of these lineages, including the Crenarchaeota (Schelert et al., 2006), Aquificae (Freedman et al., 2012), and Thermus/Deinococcus (Wang et al., 2009), varied from repression only (Schelert et al., 2006) to constitutive (Freedman et al., 2012). These observations led us to hypothesize that the evolution of mer has progressed by the sequential recruitment of functions through evolutionary time, resulting in the highly efficient and tightly regulated $\mathrm{Hg}$ detoxification mechanism that has been identified and examined in great detail among more recently evolved bacterial lineages (Barkay et al., 2003). Here, in an effort to better understand the evolution of $\mathrm{Hg}$ detoxification, we employed an integrated bioinformatic and phylogenetic approach to examine the distribution of various mer functions in all sequenced microbial genomes (as of December 2011) in order to reconstruct the evolutionary history of mer operon architecture as related to microbial evolution on Earth.

\section{MATERIALS AND METHODS mer OPERON COMPOSITION}

A total of 272 mercuric reductase (MerA) protein homologs were compiled from all completed and publically available sequence databases using the DOE IMG and the NCBI servers in December of 2011 using tblastn and MerA from Tn501 [CAA77323; (Stanisich et al., 1977)] and Sulfolobus solfataricus P2 (AAK42805; Schelert et al., 2004) as queries (Table S1 in Supplementary Material). In addition, this tabulation included several gene sequences from a number of taxa for which genomes have not been 
completed but for which the operon has been genetically characterized. All putative MerA sequences were examined manually for the presence of sequence signatures that have been experimentally shown to be essential for MerA activity (Barkay et al., 2003). These included the conserved cysteine pair at positions 207 and 212 [numbering in reference to MerA of Bacillus sp. RC607 (BAB62433)] in the redox active site, the vicinal cysteine pair at the carboxy terminus (positions 628 and 629), tyrosine at position 264 (Rennex et al., 1993), and tyrosine at position 605 for bacterial MerA and phenylalanine at position 605 for archaeal MerA (Simbahan et al., 2005). The presence of other mer gene homologs proximal to putative merA in microbial genomes was determined manually using the Neighborhood viewer on the DOE IMG server or by the Gene record function on the NCBI server. The presence of homologs of arsR, merR (divergent or convergent to the merA homolog), merP, merT, merC, merF, merE, merG, merH, merB, merD, and TRASH domain-encoding genes was tabulated (Table S1 in Supplementary Material). In addition, mer operons were screened for multiple homologs of merR and merB, elements suggestive of horizontal gene transfer (e.g., transposition function) within $\sim 10 \mathrm{Kbp}$ of $m e r A$, and were characterized as being encoded on the chromosome or on a plasmid when this information was available.

\section{PHYLOGENETIC ANALYSIS OF MerA AND 16S rDNA}

MerA protein homologs were aligned using CLUSTALX (version 2.0.8) specifying the Gonnet 250 protein substitution matrix and default gap extension and opening penalties (Larkin et al., 2007) with dihydrolipoamide dehydrogenase from Magnetospirillum magneticum AMB-1 (YP_423326), Thermus thermophilus HB27 (YP_005669), and Pseudomonas fluorescens Pf0-1 (YP_351398) serving as outgroups. $\mathrm{N}$ terminal "NmerA" sequences were trimmed from the alignment block as previously described (Barkay et al., 2010) and the phylogeny of MerA was evaluated with aBayes-PhyML (ver. 3.0.1; Anisimova et al., 2011) using the LG amino acid substitution matrix, a discrete four category gamma substitution model (gamma shape parameter $=1.078$ ), and a defined proportion of invariant sites of 0.034 , as recommended by ProtTest (version 2.4; Abascal et al., 2005). Approximate likelihood-ratio tests (aLRT) were used as an alternative to nonparametric bootstrap frequencies. A consensus phylogenetic tree was projected from 1000 aLRT permutations using FigTree (ver. 1.2.2; http://tree.bio.ed.ac.uk/UH). Trait-based analyses require a rate smoothed phylogram in order to limit biases in rapidly evolving lineages. Thus, the phylogram was rate smoothed using a penalized likelihood approach (Sanderson, 2002) as implemented by the chronopl program where a lambda smoothing parameter of 0.8 was specified over 1000 iterations. Chronopl is a part of the Ape package (ver. 3.0-3; Paradis et al., 2004) and is implemented within the base package R (ver. 2.13.1; R Development Core Team, 2010).

Representative MerP and MerT protein sequences were compiled from mer operons, aligned as described above, and their evolutionary history evaluated with the Neighbor-Joining method due to the high conservation in the sequences (i.e., low phylogenetic signal) and the short length of the alignment blocks ( $\sim 170$ positions for each) with the program MEGA5 (Tamura et al., 2011).
The consensus tree was inferred from 100 bootstrap replicates with branches with less than $50 \%$ bootstrap support collapsed. The phylogenetic distances of MerP and MerT, as computed using the Poisson correction method, is represented as the number of amino acid substitutions per site. All ambiguous positions were removed for each sequence pair.

Small subunit (SSU) rRNA genes were compiled from completed genomes that also encoded for a homolog of MerA on chromosomal DNA. SSU rRNA genes from genomes that encoded for MerA on plasmid DNA were not included in the comparison of MerA protein and SSU rRNA gene phylogenetic distances. Compiled SSU rRNA genes were aligned with CLUSTALX, specifying the IUB DNA weight matrix, and default gap opening and extension penalties with SSU rRNA genes from the eukaryotic taxa Heteromita globosa (U42447) and Trissopathes pseudotristicha isolate HAS-31 (FJ389899) serving as outgroups. The phylogeny of the SSU rRNA genes was evaluated with aBayes-PhyML (ver. 3.0.1; Anisimova et al., 2011) using the GTR amino acid substitution matrix with a discrete 4 category gamma substitution model (gamma shape parameter $=0.629$ ) and a defined proportion of invariant sites (0.149), as recommended by jModelTest (version 0.1.1; Posada, 2008). Approximate likelihood-ratio tests (aLRT) were used as an alternative to non-parametric bootstrap frequencies. The $16 \mathrm{~S}$ rRNA gene phylogram was rate smoothed as described above.

\section{STATISTICAL ANALYSES}

A Mantel regression approach was employed to evaluate the extent to which the phylogenetic dissimilarity of MerA varied with the phylogenetic dissimilarity of SSU rRNA genes. Phylocom (ver. 4.1; Webb et al., 2008) was used to generate matrices describing Rao's phylogenetic dissimilarity of the rate smoothed MerA protein phylogram and the SSU rRNA gene phylogram, as previously described (Boyd et al., 2011). The Mantel correlation coefficient and associated $p$-value for the relationship were determined using 1000 permutations, as implemented in the XLSTAT software package (ver. 2008.7.03). Pearson linear regressions were used to evaluate the co-occurrence of genes comprising mer operons. The Pearson correlation coefficient and associated $p$-value for the relationship was determined from 1000 permutations, as implemented with XLSTAT.

The individual genes that comprise the mer operon for each taxon were treated as binary traits, with a 1 and 0 indicating the presence and absence of the trait, respectively. Trait-based evolutionary methods were then applied to determine the extent to which the MerA rate smoothed phylogeny predicts the similarity in the distribution of the trait(s) among closely related taxa. The phylogenetic signal $(K$-statistic) for each trait was quantified using the program multiphylosignal within the Picante package (Kembel et al., 2010) as implemented with the base package R (ver. 2.13.1; R Development Core Team, 2010). The $K$-statistic compares the observed phylogenetic signal of a trait to the signal under a Brownian motion model of evolution on a phylogeny (Blomberg et al., 2003). Values of $K$ that are close to one imply a Brownian motion for the evolution of a trait (or some degree of phylogenetic signal) while values greater than one indicate strong phylogenetic signal for a given trait. $K$ values closer to zero or which are negative 
correspond to a random or convergent pattern of evolution for that trait. The statistical significance of $K$ was evaluated by comparing patterns of the variance of independent contrasts of the trait on a phylogeny to a null model produced by shuffling taxa labels across the tips of the phylogeny (Kembel et al., 2010).

The complexity of mer operons was also evaluated using traitbased approaches. A metric describing the complexity of mer operons was calculated by dividing the sum of the number of mer homologs encoded in an operon by 13, the total number of types of mer-associated genes retrieved in the present study. The distribution of individual mer-encoded functions and the complexity of mer operons was mapped on the rate smoothed MerA phylogeny using the Ape package (ver. 3.0-3; Paradis et al., 2004) within the base package $\mathrm{R}$.

\section{RESULTS AND DISCUSSION}

Here, we examine the evolution of the mer operon as a function of the evolutionary history of MerA. We first present the taxonomic distribution of mer in completed genomes and then follow this with a phylogenetic assessment of the evolution of MerA. Finally, we integrate new data describing the composition of mer operons into this taxonomic and phylogenetic framework and propose a paradigm for the evolution of $\mathrm{Hg}$ detoxification among prokaryotes.

\section{TAXONOMIC DISTRIBUTION OF merA}

A total of 272 MerA homologs encoding for the mercuric reductase subunit were identified in public databases as of December 2011 (Table S1 in Supplementary Material). These 272 MerA protein homologs were distributed among 246 genomes, with 23 of these genomes encoding for multiple (two or three) homologs. MerA protein homologs were identified in both archaeal as well as bacterial genomes, but were not identified in eukaryal genomes. Among the Archaea, homologs of MerA were identified among the Crenarchaeota (15/21 available genomes) and the Euryarcheota (8/34 available genomes). Among the Bacteria, homologs of MerA were prevalent among members of the Aquificae (3/7 genomes), Actinobacteria (29/91 genomes), Firmicutes (42/328 genomes), and Proteobacteria (140/517 genomes). In addition, homologs of MerA were identified in the genomes of members of the Bacteroidetes, Chloroflexi, Deinococcus/Thermus, Tenericutes, Nitrospira, and Verrucomicrobia. As we have noted before, MerA was not found in entire microbial taxa and guilds, most notably among phototrophs and Epsilon-proteobacteria, and were only rarely identified among obligate anaerobes (Barkay et al., 2010).

\section{MerA PHYLOGENY}

The phylogenetic relationships of chromosomally encoded MerA proteins were determined for use in examining patterns in the evolution of the mer operon (Figure 2). Plasmid encoded MerA proteins were excluded from this analysis, an exclusion that should have little effect on overall conclusions (see below and Figure S1 in Supplementary Material), in order to facilitate a clear demarcation of the MerA phylogeny as it relates to species (16S rRNA gene) phylogeny. Consistent with prior analyses (Barkay et al., 2010; Freedman et al., 2012), phylogenetic reconstruction of chromosomally encoded MerA reveals well supported lineages that generally

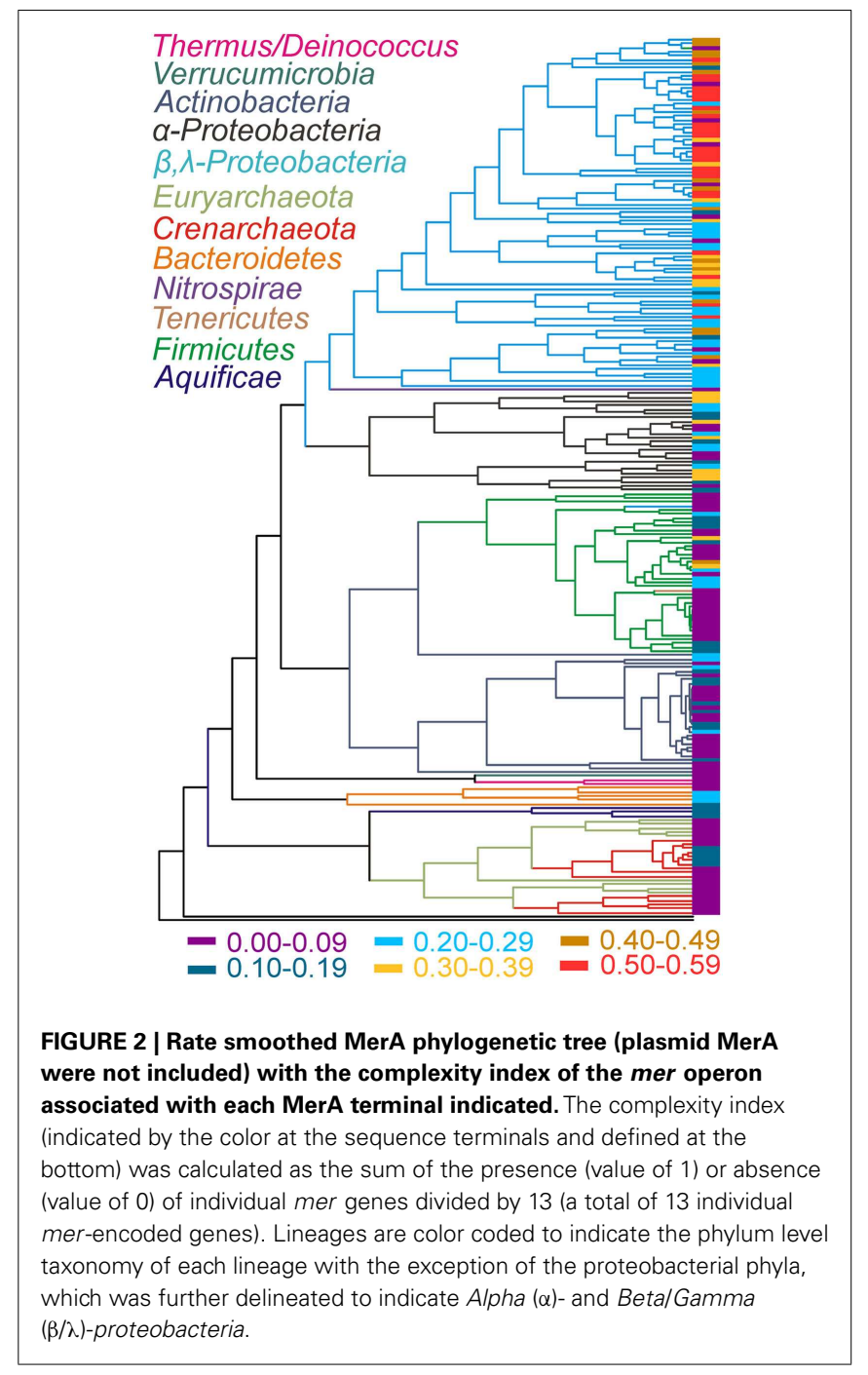

correspond with the phylum level taxonomic rank of the genomes from which they originate. The updated phylogeny consists of two large clusters, one consisting of all bacterial sequences, with the exception of Aquificae MerA. Aquificae MerA form a basal branching sub-lineage in a second large cluster that is primarily comprised of archaeal homologs. Thus, bacterial MerA is paraphyletic with respect to archaeal MerA, with Aquificae MerA branching basal to archaeal MerA indicating a bacterial origin for this enzyme. The archaeal lineage is paraphyletic with respect to the Crenarchaeota and the Euryarchaeota, with two distinct crenarchaeal and three distinct euryarchaeal lineages. Two of the euryarchaeal lineages nest the two crenarchaeal lineage. Together, the tree topology (Figure 2) may suggest that merA was first acquired within the Euryarchaeota by a lateral gene transfer (LGT) event with an ancestor of the Aquificae followed by multiple transfers between the Euryarchaeota and Crenarchaeota. Additional examples of LGT events are evident in the evolution of chromosomally encoded MerA within the more recently evolved bacteria, in particular between the Beta- and the Gamma-proteobacteria. Other examples include the cross-phylum LGT in the tenericute Acholeplasma laidlawii PG-8A that likely obtained MerA from a member of the Firmicutes, 
and the early transfer event to Nitrospirae from a member of the Proteobacteria (Figure 2). Thus, while the overall MerA phylogeny corresponds to the phylum level species phylogeny, several examples of LGT events are evident. In addition, an examination of the genomic environment flanking mer operons (only complete genomes examined) reveals evidence of recombination events as indicated by number of examples of the presence of genes encoding transposases, resolvases, inverted repeats, and phage genes (Table S1 in Supplementary Material).

We further examined the relationship between the evolution of MerA and the evolution of the organisms whose genomes encode for this function by pairwise comparisons of evolutionary distances among MerA and SSU rRNA genes, in taxa whose genomes encode for chromosomal MerA (Figure 3). A Mantel regression approach was utilized in order to identify phylogenetic barriers to gene flow among domains (Archaea and Bacteria) of organisms that encode for MerA. Here, pairwise comparisons that reveal closely related MerA genes (low phylogenetic distance) and divergent SSU rRNA genes (high phylogenetic distance) would be indicative of LGT. Broadly, the results of the Mantel test reveal that the evolution of chromosomal MerA and the evolution of the $16 \mathrm{~S}$ rRNA gene of taxa is significantly correlated (Mantel $R=0.81, p<0.01)$, suggesting an overarching role for vertical inheritance in the evolution of MerA, at least in chromosomally encoded MerA. When only bacterial MerA/16S rRNA genes are considered, the correlation is stronger (Mantel $R=0.79, p<0.01$ ) than when only archaeal MerA/16S rRNA genes are considered (Mantel $R=0.65, p<0.01$ ), a difference that could be attributed to the large evolutionary distances associated with MerA derived from the genomes of closely related Crenarchaea as depicted by several points representing short 16S rRNA gene distances with long MerA distances (Figure 3). The frequent LGT between the Archaeal phyla (see above) may account for this observation. The occurrence of LGT of merA among the Bacteria is also evident

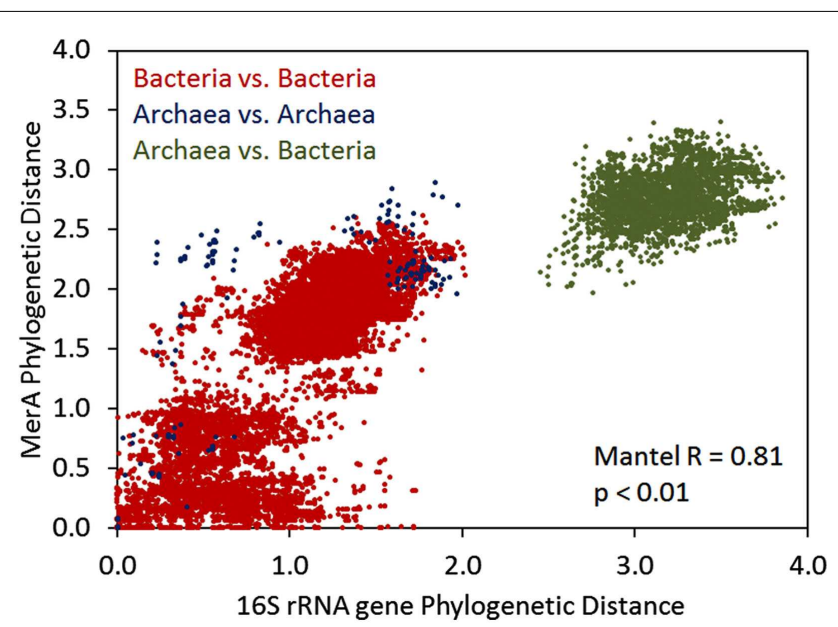

FIGURE 3 | Mantel regression of a matrix describing Rao's phylogenetic distance among MerA proteins and a matrix describing Rao's phylogenetic distance among 16S rRNA genes in all chromosomally encoded $\boldsymbol{m e r} \boldsymbol{A}$. Color overlays indicate the pairwise comparisons that are being made. in the bacterial $16 \mathrm{~S}$ rDNA vs. MerA pairwise plot with a number of points representing small MerA phylogenetic distances $[<0.1$ relative evolutionary units $(\mathrm{U})]$ and large $16 \mathrm{~S}$ rDNA phylogenetic distance $(>1.5 \mathrm{U})$, supporting previous evidence indicating that MerA in these taxa were derived by recent LGT events (Barkay et al., 2010; Lal and Lal, 2010).

\section{GENE COMPLEMENT OF mer OPERONS}

Genes flanking MerA homologs were examined for their homology to ancillary functions involved in the regulation of mer (arsR, merR, merD), Hg binding and/or transport (merT, merP, merC, merF, merE), metal sensing (TRASH), and organomercury detoxification functions ( $m e r B, \operatorname{mer} G$ ). The regulatory genes $\operatorname{ars} R$, $m e r R$, and $m e r D$ were present in 22 (8.1\% of total), $195(71.7 \%)$, and $78(28.7 \%)$ mer operons (Table 1) and with the exception of only two taxa (Bacillus cellulosilyticus DSM 2522 and Gordonia bronchialis DSM 43247), each operon encoded for either merR or $\operatorname{arsR}$ (Table S1 in Supplementary Material). merR was detected in a convergent transcriptional orientation (52 total occurrences) and more commonly in a divergent orientation (143 total occurrences), with respect to the orientation of the rest of the mer gene cluster. Genes encoding proteins implicated in $\mathrm{Hg}$ binding and/or transport [merT, merP, merC, merF, and merE (Hamlett et al., 1992)], were present in 147 (54.0\% of total), 134 (49.3\%), 56 (20.6\%), $25(9.2 \%)$, and $63(23.2 \%)$ of the mer operons examined, respectively. Genes encoding proteins with stand-alone TRASH domains, previously proposed to function in $\mathrm{Hg}$ trafficking in mer operons of Sulfolobus spp. (Ettema et al., 2003; Schelert et al., 2006) were detected in five archaeal mer operons (1.8\% of total operons examined), but were never detected in bacterial mer operons. Broad spectrum $\mathrm{Hg}$ resistance protein-encoding genes merB and merG were less frequently detected than genes encoding for narrow spectrum $\mathrm{Hg}$ resistance (e.g., merA), as indicated by the identification of these genes in only 42 (15.4\% of total), and $5(1.8 \%)$ of the mer operons examined, respectively (Table S1 in Supplementary Material).

Of the 272 mer operons identified, 53 were located on plasmids. To determine if the presence of individual mer proteins in each operon depended on whether they were plasmid or chromosomally encoded, we regressed the relative abundance of each individual mer-encoded protein in the "Plasmid Included" and "Plasmid Not Included" databases (Figure S1 in Supplementary Material). If there is no bias in the distribution of mer functions on plasmid or chromosomal DNA, then the expected slope of the regression should be 1.0 (1:1 relationship). Deviations from this 1:1 relationship would be indicative of genes that tend to be encoded on plasmid DNA or chromosomal DNA, depending on which side of the regression line they plotted. The slope of the regression analysis depicted [1.05 (Figure S1 in Supplementary Material)], was slightly higher than the expected value of the unbiased 1:1 ratio, suggestive of several proteins being more commonly encoded on plasmids. The Hg binding and/or transport functions merE, merC, merP, and merT as well as merD, a regulatory protein, all plot slightly above the 1:1 line indicating that they are more likely to be plasmid encoded. Indeed, the presence of all of these co-varied in a significant and positive relationship with their derivation from plasmid DNA (Pearson $R=0.372-0.169, p<0.005$; Table S2 in 
Table 1 | Phylogenetic signal ( $K$ value) and associated $p$-value of mer operon functions when mapped on a MerA phylologenetic tree.

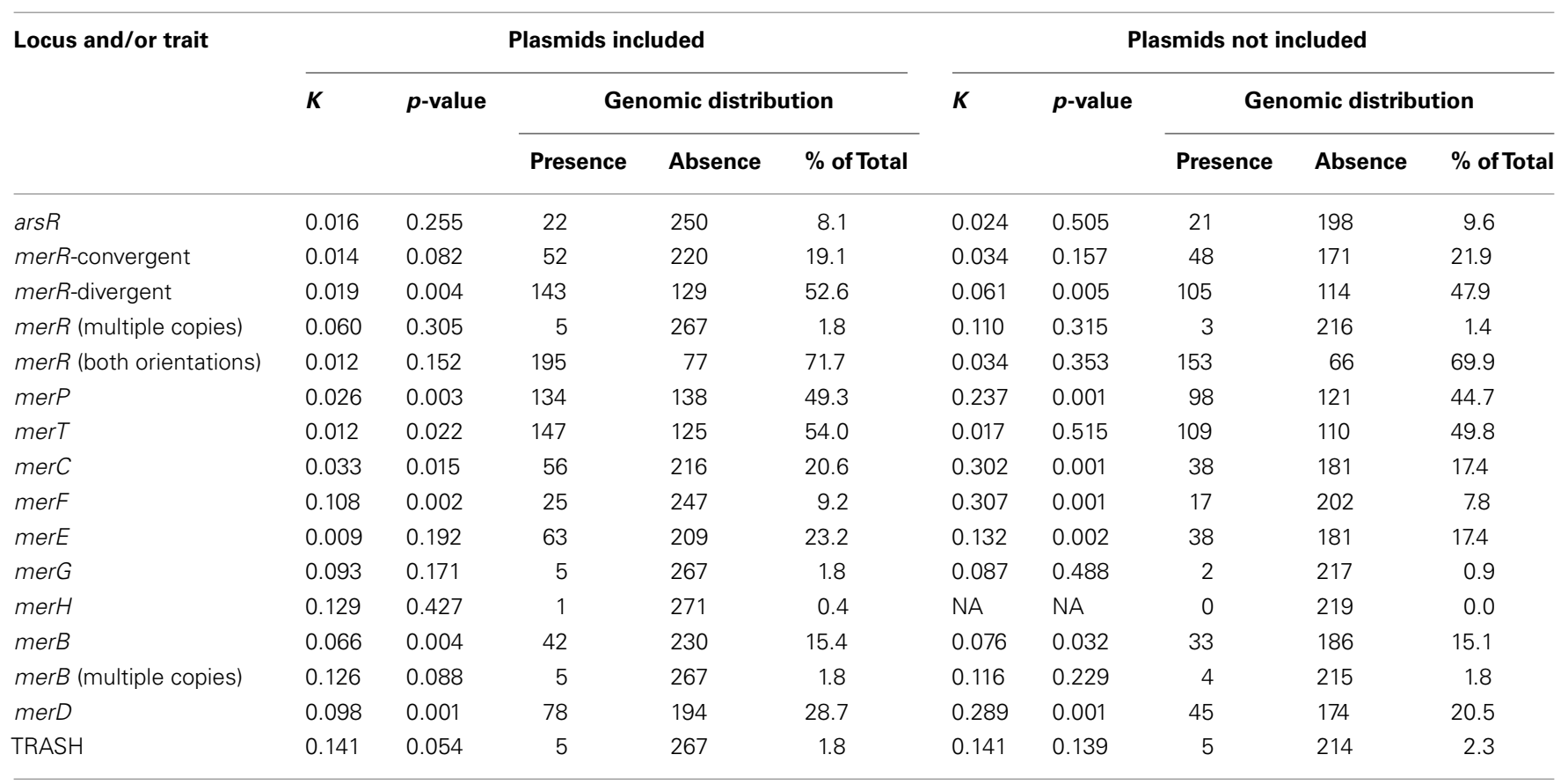

The distribution of mer functions (Presence/Absence) and \% of total mer operons encoding for a given function are indicated. Calculations were performed with all available mer operons (e.g., plasmids included) as well as with mer operons that were only encoded on chromosomes only (e.g., without plasmids).

Supplementary Material). In contrast, arsR and convergent merR (convergent orientation relative to the direction of other proximal mer genes) plot below the 1:1 line, which suggests that these proteins tend to be encoded on chromosomal DNA. Thus, the analysis suggests that several mer-encoded functions (e.g., merE, merC, $m e r P$, and $m e r T$ as well as merD) are more likely than others (arsR and merR) to be encoded on plasmids. Such observations are likely to have important ramifications for the acquisition of these genes via LGT, as discussed in more detail below. Importantly, the deviations from the 1:1 line noted above are not large, suggesting that the composition of plasmid and chromosomal mer operons are broadly similar. This observation justifies the use of either database, plasmid genes included or excluded, for further trait-based analyses as presented below.

\section{EVOLUTION OF THE mer OPERON}

Patterns in the composition of the mer operon as a function of the evolutionary history of MerA were initially examined by mapping a metric that describes its complexity (e.g., total number of mer-encoded genes) on the MerA phylogenetic tree (side bar in Figure 2). Qualitatively, this analysis indicates that the complexity of the mer operon has grown as MerA evolved, with early evolving lineages (e.g., Aquificae, Crenarchaeota, Euryarchaeota) tending to harbor less complex mer operons than more recently evolved lineages (e.g., Proteobacteria). In order to identify which genes are contributing to the increased complexity as well as to define the composition of the ancestral mer operon, the distribution of individual mer-encoded proteins was mapped on the chromosomal MerA phylogenetic tree (Figure 4, Figures S2-S13 in Supplementary Material) and the co-variation in their distribution evaluated using linear regression approaches (Table 2).
The earliest evolving bacterial mer operons, those associated with the Aquificales, are comprised of merA and the metal binding protein-encoding genes, merP (periplasmic $\mathrm{Hg}$ scavenging function) and merT (Figure 4). Intriguingly, the mer operons associated with the Archaea, which clearly acquired mer from an ancestor of the Aquificae (See above), are simpler than those of the Aquificae. The Archaeal mer operons are often comprised of only $m e r A$, and in the case of several Sulfolobus strains, an arsR regulator and a TRASH metal sensing domain protein (Schelert et al., 2006). TRASH was not found in association with any mer operons with the exception of Sulfolobus spp. and arsR was present in six of eight representative of this genus (Table S1 in Supplementary Material) leading to strong positive correlation between the two (Pearson $R=0.47, p<0.01$ ) and strongly negative correlations between TRASH and merR, merT, and merP (Pearson $R=-0.23$ to $-0.14, p=0.01-0.03$ ).

With the exception of a single euryarchaeal taxon (Ferroplasma acidarmanus fer 1 ) that encodes for a merP homolog, these transporters are absent from all taxa within the Archaea. While the absence of membrane- and periplasm-associated mer functions is expected based on the difference in cell wall structures between the domains, this finding suggests that $\operatorname{merP}$ and $\operatorname{mer} T$ (i) may not have been transferred during the LGT event that gave rise to merA in the Archaea, (ii) were purged from the genome of an ancestral Archaeon resulting in its near universal absence among extant sequenced genomes, or (iii) were acquired in the Aquificales via LGT. In order to better establish which of these possibilities is most likely, we reconstructed the evolutionary histories of MerP and MerT. Phylogenetic reconstruction of MerP (Figure S14 in Supplementary Material) and MerT (Figure S15 in Supplementary Material) revealed that those proteins derived from the Aquificales are monophyletic and branched basal to all 


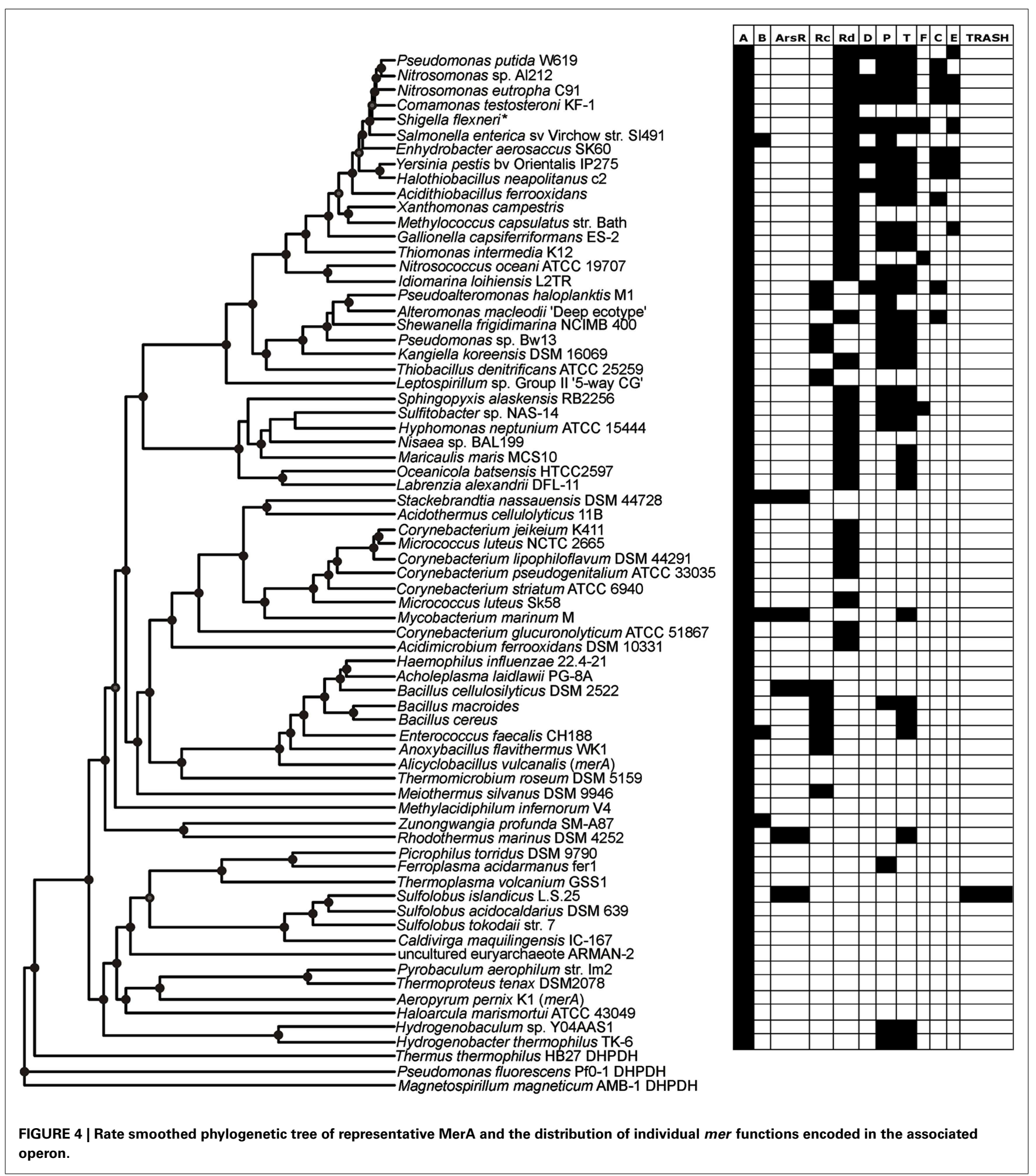

other bacterial proteins even though many instances of LGT are clearly evident in the MerP and MerT phylogenies. This observation rules out scenario (iii) above and instead suggests a vertical line of inheritance within the Aquificae and a recruitment to the mer operon early during its evolution. Importantly, MerP from
F. acidarmanus fer1 is nested among Aquificae MerP which indicates that MerP in this taxon was likely obtained through LGT; possibly the same event that led to the transfer of merA. Thus, available data suggests that the most plausible scenario is (ii) whereby merP and merT were lost following the LGT event that 
Table 2 | Pearson correlation coefficients $(R)$ and associated $p$-values indicating the co-variation in the presence/absence of individual $m e r$ operons, as assessed using linear regression.

\begin{tabular}{|c|c|c|c|c|c|c|c|c|c|c|c|c|c|}
\hline & merR(conv) & merR(div) & merR(all) & $\operatorname{ars} R$ & merP & merT & merC & merF & merE & merG & merB & merD & TRASH \\
\hline \multicolumn{14}{|c|}{ PEARSON $R$} \\
\hline $\operatorname{mer} R$ (conv) & 1.00 & -0.51 & 0.35 & -0.14 & -0.03 & -0.06 & -0.13 & -0.07 & -0.01 & 0.07 & -0.07 & -0.13 & -0.08 \\
\hline $\operatorname{merR}(\mathrm{div})$ & & 1.00 & 0.63 & -0.25 & 0.44 & 0.45 & 0.26 & 0.23 & 0.26 & 0.00 & 0.13 & 0.35 & -0.15 \\
\hline merR(all) & & & 1.00 & -0.39 & 0.45 & 0.43 & 0.17 & 0.19 & 0.27 & 0.06 & 0.08 & 0.26 & -0.23 \\
\hline $\operatorname{ars} R$ & & & & 1.00 & -0.29 & -0.17 & -0.15 & -0.09 & -0.15 & -0.03 & 0.17 & -0.17 & 0.47 \\
\hline merP & & & & & 1.00 & 0.78 & 0.36 & 0.19 & 0.39 & 0.11 & 0.06 & 0.47 & -0.14 \\
\hline $\operatorname{mer} T$ & & & & & & 1.00 & 0.32 & 0.09 & 0.32 & 0.10 & 0.14 & 0.44 & -0.15 \\
\hline merC & & & & & & & 1.00 & -0.13 & 0.40 & -0.04 & -0.06 & 0.60 & -0.07 \\
\hline merF & & & & & & & & 1.00 & 0.00 & -0.03 & -0.03 & 0.02 & -0.04 \\
\hline merE & & & & & & & & & 1.00 & 0.08 & 0.04 & 0.63 & -0.07 \\
\hline merG & & & & & & & & & & 1.00 & 0.23 & 0.19 & -0.01 \\
\hline merB & & & & & & & & & & & 1.00 & 0.07 & -0.06 \\
\hline merD & & & & & & & & & & & & 1.00 & -0.08 \\
\hline TRASH & & & & & & & & & & & & & 1.00 \\
\hline \multicolumn{14}{|l|}{$p$-VALUES } \\
\hline $\operatorname{merR}(\mathrm{conv})$ & $<0.01$ & $<0.01$ & $<0.01$ & 0.05 & 0.63 & 0.35 & 0.06 & 0.29 & 0.89 & 0.34 & 0.31 & 0.05 & 0.23 \\
\hline merR(div) & & $<0.01$ & $<0.01$ & 0.00 & $<0.01$ & $<0.01$ & $<0.01$ & $<0.01$ & $<0.01$ & 0.95 & 0.05 & $<0.01$ & 0.03 \\
\hline merR(all) & & & $<0.01$ & $<0.01$ & $<0.01$ & $<0.01$ & 0.01 & 0.00 & $<0.01$ & 0.35 & 0.23 & $<0.01$ & 0.00 \\
\hline $\operatorname{ars} R$ & & & & $<0.01$ & $<0.01$ & 0.01 & 0.03 & 0.16 & 0.03 & 0.65 & 0.01 & 0.01 & $<0.01$ \\
\hline merP & & & & & $<0.01$ & $<0.01$ & $<0.01$ & 0.01 & $<0.01$ & 0.12 & 0.40 & $<0.01$ & 0.04 \\
\hline $\operatorname{mer} T$ & & & & & & $<0.01$ & $<0.01$ & 0.20 & $<0.01$ & 0.15 & 0.04 & $<0.01$ & 0.02 \\
\hline merc & & & & & & & $<0.01$ & 0.05 & $<0.01$ & 0.52 & 0.39 & $<0.01$ & 0.30 \\
\hline merF & & & & & & & & $<0.01$ & 0.97 & 0.68 & 0.69 & 0.75 & 0.51 \\
\hline merE & & & & & & & & & $<0.01$ & 0.22 & 0.53 & $<0.01$ & 0.30 \\
\hline merG & & & & & & & & & & $<0.01$ & 0.00 & 0.01 & 0.83 \\
\hline merB & & & & & & & & & & & $<0.01$ & 0.30 & 0.34 \\
\hline merD & & & & & & & & & & & & $<0.01$ & 0.25 \\
\hline TRASH & & & & & & & & & & & & & $<0.01$ \\
\hline
\end{tabular}

Only mer operons that were encoded on a chromosome were considered. An analogous analysis with all mer operons included is presented in Table S2 in Supplementary Material.

gave rise to mer among the Archaea. This scenario is further bolstered by the previously documented central role of gene loss in the evolution of microbial genomes (Ochman et al., 2000; Morris et al., 2012). Moreover, this finding adds further support to the hypothesis that mer evolved among the Aquificae and was laterally transferred to the Archaea via interaction with an ancestral euryarchaeon.

The increased complexity of mer operons with the evolution of MerA can be attributed to the gradual addition of functions involved in the regulation of the operon by $\mathrm{Hg}, \mathrm{Hg}$ transport, and organomercury resistance (Figures 2 and 4). mer operons associated with the Aquificales lack homologs of regulatory elements (Figure 4, Figure S2 and Table S1 in Supplementary Material) and recent experimental evidence suggests that exposure to sub lethal concentrations of $\mathrm{Hg}$ did not induce further expression of merA in Hydrogenivirga sp. 128-5-R1-1 and Hydrogenobaculum sp. Y04AAS1 (Freedman et al., 2012), suggesting constitutive expression. In contrast, mer operons associated with a number of Euryarchaeota and Crenarchaeota encode for ArsR-like regulators as do mer operons associated with the Bacteroidetes [which branch basal to all bacterial MerA lineages in the large bacterial clade of the tree (Figure 2)], a number of Actinobacteria, as well as a few firmicutes (Table S1 in Supplementary Material). ArsR is a regulator that, in the absence of $\mathrm{Hg}$, represses mer expression in S. solfataricus P2 (Schelert et al., 2006) and in Streptomyces lividans (Brunker et al., 1996). ArsR is completely absent in all mer operons associated with other phyla, most notably the large Proteobacteria clades. In the Proteobacteria, as well as among most of the Firmicutes, ArsR-like regulatory elements are replaced by MerR (Figures S2, S10, and S11 in Supplementary Material). MerR represses mer operon transcription in the absence of $\mathrm{Hg}$ and induces expression in its presence (Summers, 1992; Guo et al., 2010), enhancing gene expression by several orders of magnitude in response to $\mathrm{Hg}$ exposure (Barkay et al., 2003). Finally, a second regulatory function, MerD, implicated in the down regulation of mer expression following decline in intracellular $\mathrm{Hg}$ pools (Mukhopadhyay et al., 1991 ) is only present in the Beta- and Gamma-proteobacteria, indicating a relatively recent origin for this function. As a result of the clear distribution patterns of regulatory elements among taxa, 
strong inverse correlations were observed (Table 2) between the presence/absence of arsR and merR [both orientations (Pearson $R=-0.39, p<0.01$ ) ] and $\operatorname{ars} R$ and $\operatorname{merD}$ (Pearson $R=-0.17$, $p=0.01)$ in mer operons.

Another distinction of mer operon regulation is the divergent vs. convergent orientation of merR relative to that of other mer genes. The earliest evolving MerA proteins that also encode for MerR regulatory elements are found in the Thermus/Deinococcus lineage and are transcribed convergently with merA (Wang et al., 2009). Convergently transcribed regulatory elements predominate among the Firmicutes, are rare in the Beta- and Gammaproteobacteria, and are missing among the Alpha-proteobacteria and the Actinobacteria (Table S1 in Supplementary Material). Divergently transcribed regulatory elements emerged shortly after convergent merR in the Actinobacteria, and are common in the Proteobacteria. Despite the non-conformity in the inheritance of the orientation by which merR is transcribed, that the variation is rooted relatively deep in the evolutionary history of mer is indicated by the phylum level coherence in transcription orientation. The biological meaning of convergent vs. divergent evolution is the opportunity afforded by the later to differentially express merR and functional proteins of the operon. Thus, in proteobacterial operons MerR acts as a repressor of its own transcription regardless of the presence of $\mathrm{Hg}$ while it is a repressor-activator of the rest of the divergently transcribed genes (Summers, 1992). A positive correlation (Table 2) between merD and merR-divergent (Pearson $R=0.35, p<0.01)$ and an inverse correlation between merD and merR-convergent (Pearson $R=-0.13, p=0.05$ ) reflect the later diversification of both merD and merR-divergent relative to merRconvergent. Together, it seems that the evolution of the regulation of mer operon expression has progressed from constitutive expression in the Aquificales, organisms whose natural habitats include sulfidic and often low pH springs (Huber and Eder, 2006) where geologically derived $\mathrm{Hg}$ concentrations are elevated and likely constantly present (King et al., 2006; Boyd et al., 2009), to repressed expression of operons associated with early lineages of archaeal and bacterial MerA, to an efficiently regulated system among aerobes that may be intermittently exposed to high concentrations of $\mathrm{Hg}$ in contaminated environments. This emerging paradigm optimizes the balance between the high costs of maintaining and expressing mer operon proteins and the high toxicity of $\mathrm{Hg}$ to microbiota.

A large number of proteins that are involved in metal binding and/or transport exhibit distributional patterns on the MerA tree that corroborate an overall increased complexity of the mer operon as a function of MerA evolutionary time. Genes encoding for the periplasmic $\mathrm{Hg}$ scavenging protein, MerP, and the inner membrane spanning protein, MerT, are by far the most common (Table 1; Table S1 in Supplementary Material), present in the earliest Aquificae operons, and are distributed throughout the tree (Figure 4). In contrast, genes encoding alternative transporters, MerC, MerF, MerE, and MerH, are qualitatively more prevalent in operons associated with more recently evolved MerA lineages. For example, $\operatorname{merC}$ (Figure S4 in Supplementary Material) is only present in mer operons associated with Beta- and Gamma-proteobacteria. Both merE and merF homologs exhibit a patchy distribution among the Firmicutes and Proteobacteria (Figures S6 and S7 in Supplementary
Material), but are absent in early evolving taxa. These transporters vary in the number of predicted membrane spanning domains [three in MerT, four in both MerC and MerH, and two in both MerF and MerE (Barkay et al., 2003; Lin et al., 2012)] and in substrate specificity, with MerE specific to inorganic and $\mathrm{MeHg}$ and MerT to inorganic and arylmercury (e.g., phenylmercury acetate), but not to MeHg [summarized in (Lin et al., 2012)]. Co-varying patterns of distribution of $\mathrm{Hg}$ transporters/binding functions and other mer-encoded functions (Table 2), reflect both known interactions [e.g., the positive correlation between MerP and MerT (Pearson $R=0.78, p<0.001)$ ] and with the relative time of their appearance during the evolution of MerA, as exhibited by strong and inverse correlations between ArsR and all transport/binding functions (Pearson $R=-0.29$ to $-0.14, p<0.01-0.03$ ).

The distribution of genes that encode proteins involved in broad spectrum $\mathrm{Hg}$ resistance, and which are related to organomercurial degradation (MerB, MerG), also exhibited a patchy distribution with respect to the evolution of MerA. merB genes were identified among members of the Bacteroidetes, Actinobacteria, Firmicutes, and Proteobacteria. The merG gene, which encodes a protein thought to be involved in reducing cellular permeability of phenylmercury (Kiyono and Pan-Hou, 1999), was only identified in five operons, three of which originated in pseudomonads with the remaining two detected on plasmids that were directly isolated from soil (Table S1 in Supplementary Material). merG gene homologs were never identified in mer operons that did not also encode for merB genes (hence the strong positive correlation between the two, Pearson $R=0.23, p=0.01$ ), consistent with their documented function in attenuating the toxicity of organomercurial compounds (Kiyono and Pan-Hou, 1999). Importantly, the recruitment of MerG protein-encoding genes to the mer operon and the enhanced protection that this gives cells to the toxic effects of organomercurials, coupled with the fact that mer $G$ genes are only present in more recently evolved taxa, suggests this to be another example of how the mer detoxification system has been refined through evolutionary time and possibly in response to the release of anthropogenic organomercury contaminants.

\section{THE ROLE OF PLASMIDS IN THE EVOLUTION OF THE mer OPERON}

The qualitative patterns observed in the distribution of mer functions in chromosomally encoded genes, as outlined above, were quantified using trait-based modeling tools. Here, each gene was treated as a "trait" and the dispersion of traits was mapped on the rate smoothed MerA phylogram and were then quantified. The $K$ statistic compares the observed signal of a trait to the signal under a Brownian motion model of evolution on a phylogeny (Blomberg et al., 2003). Values of $K$ that are close to one imply a Brownian motion for the evolution of a trait (or some degree of phylogenetic signal) while values greater than one indicate strong phylogenetic signal for a given trait. $K$ values closer to zero or which are negative correspond to a random or convergent pattern of evolution for that trait. Variation in the $K$-statistic when only chromosomal mer operons were considered as compared to values obtained when all mer operons (chromosomal and plasmid-origin) were considered, are attributable to plasmids obscuring vertical patterns of inheritance. In such a scenario, decreases in $K$ are indicative 


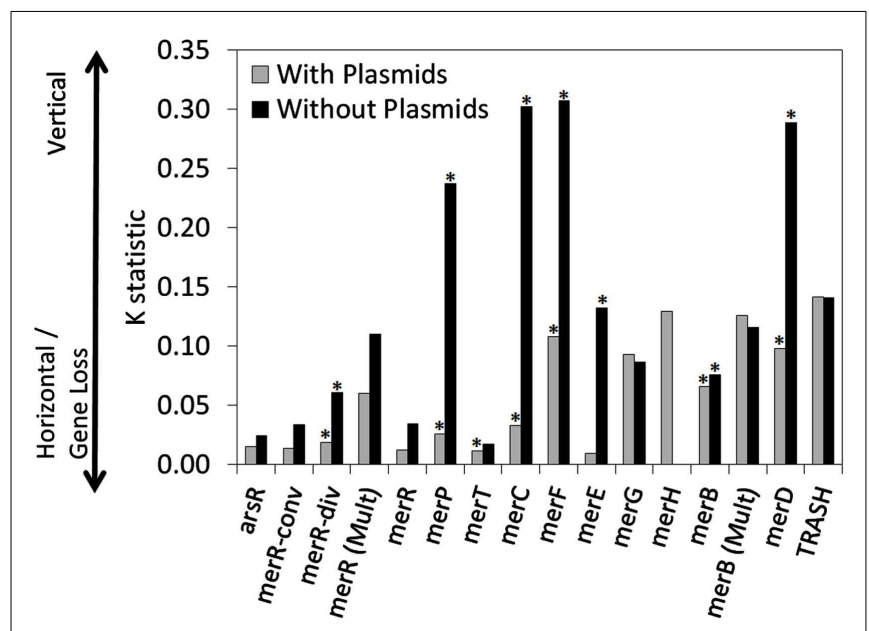

FIGURE 5 | The $\boldsymbol{K}$-statistic associated with individual mer functions, when mapped on the rate smoothed MerA phylogenetic tree. $K$-statistics were calculated for mer functions encoded on operons that were either chromosomal encoded (e.g., without plasmids) or that were chromosomal and plasmid encoded (with plasmids). $K$ values that were statistically significant ( $p$-value $<0.05$ ) are indicated with an asterisk. Low $K$ values are suggestive of a strong tendency for the gene to be subjected to LGT or gene loss. In contrast, high $K$ values are suggestive of vertical transmission of the gene.

of plasmid-based LGT events decreasing the signal for vertical inheritance.

A number of chromosomal mer-encoded genes exhibited statistically significant and positive $K$ values, including merP $(K=0.237, p<0.001)$, merC $(K=0.302, p<0.001), \operatorname{merF}$ $(K=0.307, p<0.001)$ and $\operatorname{merD}(K=0.289, p<0.001$; Table 1 ; Figure 5), suggesting a tendency for these genes to be transferred vertically through chromosome-based replication and diversification. However, when the phylogenetic distribution of plasmidbased mer functions was also included in the analysis, the $K$ statistic decreased dramatically for these functions $(K=0.026$, $0.033,0.108,0.098$, respectively). This large decrease in the $K$ statistic indicates that the pattern of vertical inheritance of these genes was obscured by the inclusion of plasmid-based sequences, indicating a prevalent role for plasmids in the dispersion of mer operons and individual mer functions across phylogenetic boundaries. Indeed, a large decrease in the $K$-statistic was also observed in the case of merR (conv), merR (div), merR (total), merT, $m e r E$, and $\operatorname{mer} B$, with several of these decreases being statistically significant [merR (div), merT, merE, and merB]. Due to the fact that the $K$-statistic is sensitive to the phylogenetic distance between and among organisms that share or lack a given trait (i.e., mer-encoded gene; Blomberg et al., 2003), those genes with $K$ values that underwent the most significant decreases when plasmid-based mer were included in the analysis (Figure 5) can be considered to be those which are least sensitive to phylogenetic boundaries in their dispersion. The mer genes that underwent the largest decrease in the $K$-statistic were the genes encoding for proteins involved in $\mathrm{Hg}$ transport/binding $\operatorname{mer} C$, merP, merF, and merE, and regulation, merD. Qualitative examinations of the distribution of these genes on the rate smoothed
MerA phylogeny (Figures S4-S7 and S9 in Supplementary Material, respectively) reveal a limited and patchy distribution of these genes within one or two closely related phyla. Such observations, coupled with the results of the differential $K$-statistic when plasmids are included/excluded, suggests that plasmid-based LGT is a likely mechanism influencing the evolution of the mer operon.

None of the $K$ values obtained in the present analyses of individual mer operon functions (both plasmid encoded mer operons included and excluded), when mapped on the MerA phylogram, yield particularly high $K$ values that would be indicative of an overall pattern of vertical inheritance, with minimal influence from LGT. This is in stark contrast to previous analyses of the evolution of nitrogenase, which converts dinitrogen gas to ammonia (Rubio and Ludden, 2008), as well as Suf proteins, which are involved in iron sulfide cluster biosynthesis (Outten et al., 2004), where strong evidence ( $K$ values $>1.0$ ) for vertical descent in ancillary and core functions has been previously observed (Eric S. Boyd, unpublished data). This observation, suggest that although the evolution of MerA, the core of the mercury detoxification mechanism, is likely to have occurred primarily by vertical descent, a number of the ancillary functions have been subjected to extensive LGT or gene loss. This is likely due to the high energetic costs of maintaining ancillary functions that are not required for enzymatic activity, resulting in a strong selective pressure to purge genomes of ancillary functions that lead to an overall decrease in organismal fitness (Morris et al., 2012).

\section{THE ROLE OF LGT IN mer OPERON EVOLUTION}

In contrast to ancillary mer-encoded functions, which appear to be subject to extensive gene loss and transfer events, evidence for an extenstive role of LGT in the evolution of MerA is relatively scarce. The relative scarcity for LGT of MerA, which is subject to a strong positive selection (Gogarten et al., 2001), is an enigma which we previously attributed to the co-varying effects of environmental conditions on $\mathrm{Hg}$ bioavailability and microbial species distribution (e.g., tendency for phylogenetically related taxa to inhabit similar ecological niches; Barkay et al., 2010). In addition to selective pressure, several other processes affect the extent of LGT [reviewed by Thomas and Nielsen (2005)] and the integration and stable inheritance of the transferred genetic material in the recipient genome [reviewed by Boto (2009)], the consequences of which are detected by phylogenetic reconstructions (Ragan, 2001). The phylogenetic distance between taxa has been proposed as a modulator of inheritance by LGT so that while rare events of cross-domain transfer are known, the frequency of LGT events increases as the phylogenetic distance between donor and recipient declines (Gogarten et al., 2001; Boto, 2009). When tested, the relative frequency of within phylum as compared to cross-phylum LGT has been shown to be dependent on the phyla considered (Zhaxybayeva et al., 2006, 2009). Careful examination of the Alpha-proteobacteria MerA clade, for which our current and previous analyses indicate an almost full congruence between the gene and the species trees [Figures 2 and 4; (Freedman et al., 2012) Figure S1 in Supplementary Material; (Barkay et al., 2010) Figure 1)], however, did reveal several cross-order transfers among marine, and between soil and 
marine, strains. The other congruent cluster, the Actinobacteria, consisted in its entirety of the order Actinomycetales. We therefore conclude that while LGT seems a forceful process leading to horizontal spread of mer genes as evident by frequent plasmid carriage (Figure 5) and the genomic proximity to signatures of past LGT events [e.g., transposases, resolvases (Table S1 in Supplementary Material)], the evolutionary signals of such events on the evolution of MerA have ameliorated due to post transfer selection exerted by the environment as well as by the intracellular constraints on mer functions, e.g., MerA dependence on intracellular redox buffering (Ledwidge et al., 2005) and interactions with membrane constituents of $\mathrm{Hg}$ transporters (Barkay et al., 2003).

\section{CONCLUSIONS AND RELEVANCE TO MERCURY BIOREMEDIATION}

The $\mathrm{Hg}$ resistance system, as present in a large number of extant microorganisms, is a modular system (Liebert et al., 2000) that has been assembled over evolutionary time spans by strong selection and enhanced fitness in environments with varying patterns of exposure to $\mathrm{Hg}$. Initially limited to geothermal environments where exposure to geological sources of $\mathrm{Hg}$ has driven the derivation of MerA likely through ancient gene duplication and subsequent mutations of another flavin-nucleotide disulfide oxidoreductase (Pullikuth and Gill, 1997), mer operons have gradually increased their gene complements and functional diversity (Figures 2 and 4), in particular with respect to the efficiency of regulatory control on gene expression. A simple constitutively expressed system affording resistance in environments with constant exposure to $\mathrm{Hg}$ has become a tightly regulated efficient $\mathrm{Hg}$ detoxification machine in diverse environments.

The database studied here was established based on annotation of ORF's proximal to merA as mer gene homologs and it is therefore limited to functions previously identified to play a role in $\mathrm{Hg}$ detoxification. A limitation of this approach is the potential omission of genes, and the functions they specify, that are a part of uncharacterized mer operons. During our genome surveys we noticed the presence of many gene homologs, some related to metal sensing and trafficking or to cysteine biosynthesis, embedded within putative mer operons [for examples see Table S1 in Supplementary Material in Barkay et al. (2010)]. These homologs, especially those shown to be co-transcribed with mer $A$ in response to $\mathrm{Hg}$ (II) exposure (Schelert et al., 2006; Wang et al., 2009), may play various uncharacterized roles in the response to $\mathrm{Hg}$ toxicity. It is likely that once characterized, these traits will expand our understanding of the mer paradigm and its evolution.

With the exception of the widespread oxygenation of the Earth's biosphere which increased the bioavailability of $\mathrm{Hg}$ in its most oxidized form (Barkay et al., 2010), it is currently not possible to relate events in mer operon evolution to an absolute time frame and/or specific events in Earth history. It is, however, plausible that industrial activities resulting in the release of $\mathrm{Hg}$ to natural waters and soils have accelerated the evolution of mer systems due to everincreasing selective pressure associate with the use of mercurial compounds as catalysts, disinfectants, pesticides, and herbicides (Liu et al., 2012). The emerging efficient detoxification machine that converts highly toxic $\mathrm{Hg}$ (II) and organomercury compounds to less reactive and volatile $\mathrm{Hg}(0)$ has provided important tools in bioremediation and the environmental management of $\mathrm{Hg}$ contamination. These applications are only possible thanks to the detailed understanding of how mer systems function and evolve in response to the ever-increasing challenge of $\mathrm{Hg}$ to life.

\section{ACKNOWLEDGMENTS}

This work was supported by National Science Foundation grants EAR-1123689, EAR-1024614, and PIRE-0968421 (Eric S. Boyd) and by the Office of Science (BER), U.S. Department of Energy Grants No. DE-FG02-08ER64544 and DE-SC0007051 (Tamar Barkay).

\section{SUPPLEMENTARY MATERIALS}

The Supplementary Material for this article can be found online at http://www.frontiersin.org/Microbiotechnology,_Ecotoxicology_ and_Bioremediation/10.3389/fmicb.2012.00349/abstract

Table S1 | mer operons included in this study.

Table S2 | Pearson correlation coefficients (R) and associated $p$-values indicating the co-variation in the presence/absence of individual mer operons, as assessed using linear regression. Only mer operons that were encoded on a chromosome were considered. Abbreviations: $\operatorname{merR}(\mathrm{c})$, merR-convergent; $\operatorname{mer} R(\mathrm{~d}), \operatorname{mer} R$-divergent; $\operatorname{mer} R(\mathrm{a}), \operatorname{mer} R$ - both convergent and divergent; PI, plasmid encoded.

Figure S1 | Plot of the percent of taxa that encode for individual mer functions when all mer operons are included (chromosomal + plasmid) and the percent of taxa that encode for individual mer functions when only chromosomal mer operons are included (no plasmids). A 1:1 line is plotted as well. Genes that plot above the 1:1 line are suggestive of having a higher tendency to be encoded in plasmid-based mer operons and are more likely to be subjected to LGT than genes that plot below this line.

Figure S2 |The taxonomic distribution of ars $R$ mapped on the MerA phylogenetic tree, as indicated by blue crosses to the right of the sequence terminal. Phylum level taxonomic rankings are overlaid by color on each lineage.

Figure S3 | The taxonomic distribution of merB mapped on the MerA phylogenetic tree, as indicated by blue crosses to the right of the sequence terminal. Phylum level taxonomic rankings are overlaid by color on each lineage.

Figure S4 |The taxonomic distribution of merC mapped on the MerA phylogenetic tree, as indicated by blue crosses to the right of the sequence terminal. Phylum level taxonomic rankings are overlaid by color on each lineage.

Figure S5 | The taxonomic distribution of merD mapped on the MerA phylogenetic tree, as indicated by blue crosses to the right of the sequence terminal. Phylum level taxonomic rankings are overlaid by color on each lineage.

Figure S6 |The taxonomic distribution of merE mapped on the MerA phylogenetic tree, as indicated by blue crosses to the right of the sequence terminal. Phylum level taxonomic rankings are overlaid by color on each lineage.

Figure S7 |The taxonomic distribution of merF mapped on the MerA phylogenetic tree, as indicated by blue crosses to the right of the sequence terminal. Phylum level taxonomic rankings are overlaid by color on each lineage.

Figure S8 |The taxonomic distribution of merG mapped on the MerA phylogenetic tree, as indicated by blue crosses to the right of the sequence terminal. Phylum level taxonomic rankings are overlaid by color on each lineage. 
Figure S9 | The taxonomic distribution of merP mapped on the MerA phylogenetic tree, as indicated by blue crosses to the right of the sequence terminal. Phylum level taxonomic rankings are overlaid by color on each lineage.

Figure S10 |The taxonomic distribution of merR (divergent orientation) mapped on the MerA phylogenetic tree, as indicated by blue crosses to the right of the sequence terminal. Phylum level taxonomic rankings are overlaid by color on each lineage.

Figure S11 |The taxonomic distribution of merR (convergent orientation) mapped on the MerA phylogenetic tree, as indicated by blue crosses to the right of the sequence terminal. Phylum level taxonomic rankings are overlaid by color on each lineage.

Figure S12 |The taxonomic distribution of merT mapped on the MerA phylogenetic tree, as indicated by blue crosses to the right of the sequence

\section{REFERENCES}

Abascal, F., Zardoya, R., and Posada, D. (2005). ProtTest: selection of best-fit models of protein evolution. Bioinformatics 21, 2104-2105.

Anisimova, M., Gil, M., Dufayard, J. F., Dessimoz, C., and Gascuel, O. (2011). Survey of branch support methods demonstrates accuracy, power, and robustness of fast likelihood-based approximation schemes. Syst. Biol. 60, 685-699.

Barkay, T. (1987). Adaptation of aquatic microbial communities to $\mathrm{Hg}^{2+}$ stress. Appl. Environ. Microbiol. 53, 2725-2732.

Barkay, T., Kritee, K., Boyd, E., and Geesey, G. (2010). A thermophilic bacterial origin and subsequent constraints by redox, light and salinity on the evolution of the microbial mercuric reductase. Environ. Microbiol. 12, 2904-2917.

Barkay, T., Miller, S. M., and Summers, A. O. (2003). Bacterial mercury resistance from atoms to ecosystems. FEMS Microbiol. Rev. 27, 355-384.

Blomberg, S. P., Garland, T. Jr., and Ives, A. R. (2003). Testing for phylogenetic signal in comparative data: behavioral traits are more labile. Evolution 57, 717-745.

Boto, L. (2009). Horizontal gene transfer in evolution: facts and challenges. Proc. Biol. Sci. 277, 819-827.

Boyd, E. S., Hamilton, T. L., and Peters, J. W. (2011). An alternative path for the evolution of biological nitrogen fixation. Front. Microbiol. 2:205. doi:10.3389/fmicb.2011.00205

Boyd, E. S., King, S. A., Tomberline, J. K., Nordstrom, D. K., Krabbenhoft, D. P., Barkay, T., et al. (2009). Methylmercury enters an aquatic food web through acidophilic microbial mats in Yellowstone National Park, Wyoming. Environ. Microbiol. 11, 950-959.

Brunker, P., Rother, D., Sedlmeier, R., Klein, J., Mattes, R., and
Altenbuchner, J. (1996). Regulation of the operon responsible for broad-spectrum mercury resistance in Streptomyces lividans 1326. Mol. Gen. Genet. 251, 307-315.

Clarkson, T. W., and Magos, L. (2006) The toxicology of mercury and its chemical compounds. Crit. Rev. Toxicol. 36, 609-662.

Dietz, R., Outridge, P. M., and Hobson, K. A. (2009). Anthropogenic contributions to mercury levels in presentday Arctic animals - a review. Sci. Total Environ. 407, 6120-6131.

Ettema, T. J. G., Huynen, M. A., De Vos, W. M., and Van Der Oost, J. (2003). TRASH: a novel metalbinding domain predicted to be involved in heavy-metal sensing, trafficking and resistance. Trends Biochem. Sci. 28, 170-173.

Freedman, Z., Zhu, C., and Barkay, T. (2012). Mercury resistance, mercuric reductase activities and expression among chemotrophic thermophilic Aquificae. Appl. Environ. Microbiol. 78, 6568-6575.

Gogarten, J. P., Doolittle, W. F., and Lawrence, J. G. (2001). Prokaryotic evolution in light of gene transfer. Mol. Biol. Evol. 19, 2226-2238.

Guo, H. B., Johs, A., Parks, J. M., Olliff, L., Miller, S. M., Summers, A. O., et al. (2010). Structure and conformational dynamics of the metalloregulator MerR upon binding of $\mathrm{Hg}(\mathrm{II})$. J. Mol. Biol. 398, 555-568.

Hamlett, N. V., Landale, E. C., Davis, B. H., and Summers, A. O. (1992). Roles of the Tn21 merT, merP, and merC gene products in mercury resistance and mercury binding. $J$. Bacteriol. 174, 6377-6385.

Huber, H., and Eder, W. (2006). Aquificales. Prokaryotes 7, 925-938.

Kembel, S. W., Cowan, P. D., Helmus, M. R., Cornwell, W. K., Morlon, H., Ackerly, D. D., et al. (2010). Picante: $\mathrm{R}$ tools for integrating

terminal. Phylum level taxonomic rankings are overlaid by color on each lineage.

Figure S13 |The taxonomic distribution of the TRASH metal binding domain protein mapped on the MerA phylogenetic tree, as indicated by blue crosses to the right of the sequence terminal. Phylum level taxonomic rankings are overlaid by color on each lineage.

Figure S14 | Phylogenetic reconstruction of MerP, as determined using the Neighbor-Joining method. The tree is rooted with a paralogous pair of proteins that are putatively involved in heavy metal transport/detoxification.

Figure S15 | Phylogenetic reconstruction of MerT, as determined using the Neighbor-Joining method. The tree is rooted with a paralogous pair of proteins that are involved in binding copper.

phylogenies and ecology. Bioinformatics 26, 1463-1464.

King, S. A., Behnke, S., Slack, K. Krabbenhoft, D. P., Nordstrom, D. K., Burr, M. D., et al. (2006). Mercury in water and biomass of microbial communities in hot springs of Yellowstone National Park, USA. Appl. Geochem. 21, 1868-1879.

Kiyono, M., and Pan-Hou, H. (1999). The merG gene product is involved in phenylmercury resistance in Pseudomonas strain K-62. J. Bacteriol. 181, 726-730.

Kritee, K., Blum, J. D., Johnson, M. W., Bergquist, B. A., and Barkay, T. (2007). Mercury stable isotope fractionation during reduction of $\mathrm{Hg}$ (II) to $\mathrm{Hg}(0)$ by mercury resistant microorganisms. Environ. Sci. Technol. 41, 1889-1895.

Lal, D., and Lal, R. (2010). Evolution of mercuric reductase (merA) gene: a case of horizontal gene transfer. Mikrobiologiia 79, 524-531.

Larkin, M. A., Blackshields, G., Brown, N. P., Chenna, R., Mcgettigan, P. A., Mcwilliam, H., et al. (2007). Clustal $\mathrm{W}$ and Clustal X version 2.0. Bioinformatics 23, 2947-2948.

Ledwidge, R., Patel, B., Dong, A., Fiedler, D., Falkowski, M., Zelikova, J., et al. (2005). NmerA, the metal binding domain of mercuric ion reductase, removes $\mathrm{Hg}^{2+}$ from proteins, delivers it to the catalytic core, and protects cells under glutathionedepleted conditions. Biochemistry 44, 11402-11416.

Liebert, C. A., Watson, A. L., and Summers, A. O. (2000). The quality of merC, a module of the mer mosaic. J. Mol. Evol. 51, 607-622.

Lin, C.-C., Yee, N., and Barkay, T. (2012). "Microbial transformations in the mercury cycle," in Environmental Chemistry and Toxicology of Mercury, eds C. Liu, C. Yong and N. O'driscoll (Hoboken, NJ: John Wiley \& Sons, Inc.), 155-191.
Liu, G., Cai, Y., O’driscoll, N., Feng, Z., and Jiang, G. (2012). "Overview of mercury in the environment," in Environmental Chemistry and Toxicology of Mercury, eds C. Liu, C. Yong and N. O'driscoll (Hoboken, NJ: John Wiley \& Sons, Inc.), $1-12$.

Morris, J. J., Lenski, R. E., and Zinser, R. E. (2012). The Black Queen Hypothesis: evolution of dependencies through adaptive gene loss. MBio 3, e00036-12.

Mukhopadhyay, D., Yu, H. R., Nucifora, G., and Misra, T. K. (1991). Purification and functional characterization of MerD. A coregulator of the mercury resistance operon in gramnegative bacteria. J. Biol. Chem. 266, 18538-18542.

Nies, D. H. (2003). Efflux-mediated heavy metal resistance in prokaryotes. FEMS Microbiol. Rev. 27, 313-339.

Ochman, H., Lawrence, J. G., and Groisman, E. A. (2000). Lateral gene transfer and the nature of bacterial innovation. Nature 405, 299-304.

Osborn, A. M., Bruce, K. D., Strike, P., and Ritchie, D. A. (1997). Distribution, diversity and evolution of the bacterial mercury resistance (mer) operon. FEMS Microbiol. Rev. 19, 239-262.

Outten, F. W., Djaman, O., and Storz, G. (2004). A sufoperon requirement for $\mathrm{Fe}-\mathrm{S}$ cluster assembly during iron starvation in Escherichia coli. Mol. Microbiol. 52, 861-872.

Paradis, E., Claude, J., and Strimmer, K. (2004). APE: analyses of phylogenetics and evolution in $\mathrm{R}$ language. Bioinformatics 20, 289-290.

Pirrone, N., Cinnirella, S., Feng, X. Finkelman, R. B., Friedli, H. R., Leaner, J., et al. (2010). Global mercury emissions to the atmosphere from anthropogenic and natural sources. Atmos. Chem. Phys. 10, 5951-5964. 
Posada, D. (2008). jModelTest: phylogenetic model averaging. Mol. Biol. Evol. 25, 1253-1256.

Pullikuth, A. K., and Gill, S. S. (1997). Primary structure of an invertebrate dihydrolipoamide dehydrogenase with phylogenetic relationship to vertebrate and bacterial disulfide oxidoreductases. Gene 200, 163-172.

R Development Core Team. (2010). R: A Language and Environment for Statistical Computing. Vienna: R Foundation for Statistical Computing.

Ragan, M. A. (2001). Detection of lateral gene transfer among microbial genomes. Curr. Opin. Genet. Dev. 11, 620-626.

Rennex, D., Cummings, R. T., Pickett, M., Walsh, C. T., and Bradley, M. (1993). Role of tyrosine residues in $\mathrm{Hg}(\mathrm{II})$ detoxification by mercuric reductase from Bacillus sp. strain RC607. Biochemistry 32, 7475-7478.

Rimmer, C. C., Miller, E. K., McFarland, K. P., Taylor, R. J., and Faccio, S. D. (2010). Mercury bioaccumulation and trophic transfer in the terrestrial food web of a montane forest. Ecotoxicology 19, 697-709.

Rubio, L. M., and Ludden, P. W. (2008). Biosynthesis of the ironmolybdenum cofactor of nitrogenase. Annu. Rev. Microbiol. 62, 93-111.

Sanderson, M. J. (2002). Estimating absolute rates of molecular evolution and divergence times: a penalized likelihood approach. Mol. Biol. Evol. 19, 101-109.

Schaefer, J. K., Yagi, J., Reinfelder, J., Cardona-Marek, T., Ellickson, K., Tel-Or, S., et al. (2004). The role of the bacterial organomercury lyase (MerB) in controlling methylmercury accumulation in mercury contaminated natural waters. Environ. Sci. Technol. 34, 4304-4311.

Schelert, J., Dixit, V., Hoang, V., Simbahan, J., Drozda, M., and Blum, P. (2004). Occurrence and characterization of mercury resistance in the hyperthermophilic archaeon Sulfolobus solfataricus by use of gene disruption. J. Bacteriol. 186, 427-437.

Schelert, J., Drozda, M., Dixit, V., Dillman, A., and Blum, P. (2006). Regulation of mercury resistance in the crenarchaeote Sulfolobus solfataricus. J. Bacteriol. 188, 7141-7150.

Selifonova, O., Burlage, R., and Barkay, T. (1993). Bioluminescent sensors for detection of bioavailable $\mathrm{Hg}(\mathrm{II})$ in the environment. Appl. Environ. Microbiol. 59, 3083-3090.

Simbahan, J., Kurth, E., Schelert, J., Dillman, A., Moriyama, E., Jovanovich, S., et al. (2005). Community analysis of a mercury hot spring supports occurrence of domainspecific forms of mercuric reductase. Appl. Environ. Microbiol. 71, 8836-8845.

Stanisich, V.A., Bennett, P. M., and Richmond, M. H. (1977). Characterization of a translocation unit encoding resistance to mercuric ions that occurs on a nonconjugative plasmid in Pseudomonas aeruginosa. J. Bacteriol. 129, 1227-1233.

Summers, A. O. (1992). Untwist and shout: a heavy metal-responsive transcriptional regulator. J. Bacteriol. 174, 3097-3101.
Tamura, K., Peterson, D., Peterson, N., Stecher, G., Nei, M., and Kumar, S. (2011). MEGA5: molecular evolutionary genetics analysis using maximum likelihood, evolutionary distance, and maximum parsimony methods. Mol. Biol. Evol. 28 2731-2739.

Thomas, C. M., and Nielsen, K. M. (2005). Mechanisms of, and barriers to, horizontal gene transfer between bacteria. Nat. Rev. Microbiol. 3, 711-721.

Virta, M., Lampinen, J., and Karp, M. (1995). A luminescence-based mercury biosensor. Anal. Chem. 67, 667-669.

Wagner-Döbler, I. (2003). Pilot plant for bioremediation of mercurycontaining industrial wastewater. Appl. Environ. Microbiol. 62 124-133.

Wang, Y., Freedman, Z., Lu-Irving, P., Kaletsky, R., and Barkay, T. (2009). An initial characterization of the mercury resistance (mer) system of the thermophilic bacterium Thermus thermophilus HB27. FEMS Microbiol. Ecol. 67, 118-129.

Watras, C. J., Back, R. C., Halvorsen, S., Hudson, R. J. M., Morrison, K. A. and Wente, S. P. (1998). Bioaccumulation of mercury in pelagic freshwater food webs. Sci. Total Environ. 219, 183-208.

Webb, C. O., Ackerly, D. D., and Kembel, S. W. (2008). Phylocom: software for the analysis of phylogenetic community structure and character evolution. Bioinformatics 24 2098-2100.

Zhaxybayeva, O., Gogarten, J. P., Charlebois, R. L., Doolittle, W. F., and Papke, R. T. (2006). Phylogenetic analyses of cyanobacterial genomes: quantification of horizontal gene transfer events. Genome Res. 16, 1099-1108.

Zhaxybayeva, O., Swithers, K. S., Lapierre, P., Fournier, G. P., Bickhart, D. M., Deboy, et al. (2009). On the chimeric nature, thermophilic origin, and phylogenetic placement of the Thermotogales. Proc. Natl. Acad. Sci. U.S.A. 106, 5865-5870.

Conflict of Interest Statement: The authors declare that the research was conducted in the absence of any commercial or financial relationships that could be construed as a potential conflict of interest.

Received: 27 July 2012; paper pending published: 27 August 2012; accepted: 11 September 2012; published online: 08 October 2012.

Citation: Boyd ES and Barkay $T$ (2012) The mercury resistance operon: from an origin in a geothermal environment to an efficient detoxification machine. Front. Microbio. 3:349. doi: 10.3389/fmicb.2012.00349

This article was submitted to Frontiers in Microbiotechnology, Ecotoxicology and Bioremediation, a specialty of Frontiers in Microbiology.

Copyright (C) 2012 Boyd and Barkay. This is an open-access article distributed under the terms of the Creative Commons Attribution License, which permits use, distribution and reproduction in other forums, provided the original authors and source are credited and subject to any copyright notices concerning any third-party graphics etc. 